\title{
Investigating Off-shell Stability of Anti-de Sitter Space in String Theory
}

\author{
V Suneeta 1 \\ Dept of Mathematical and Statistical Sciences \\ and \\ The Applied Mathematics Institute, \\ University of Alberta, Edmonton, AB, Canada T6G 2G1.
}

\begin{abstract}
We propose an investigation of stability of vacua in string theory by studying their stability with respect to a (suitable) world-sheet renormalization group (RG) flow. We prove geometric stability of (Euclidean) anti-de Sitter (AdS) space (i.e., $\mathbf{H}^{n}$ ) with respect to the simplest RG flow in closed string theory, the Ricci flow. AdS space is not a fixed point of Ricci flow. We therefore choose an appropriate flow for which it is a fixed point, prove a linear stability result for AdS space with respect to this flow, and then show this implies its geometric stability with respect to Ricci flow. The techniques used can be generalized to RG flows involving other fields. We also discuss tools from the mathematics of geometric flows that can be used to study stability of string vacua.
\end{abstract}

\footnotetext{
${ }^{1}$ vardarajan@math.ualberta.ca
} 


\section{Introduction}

It has been conjectured that off-shell processes in string theory such as tachyon condensation are described qualitatively by solutions to RG flows of world-sheet sigma models (see [1] for a review). This conjecture is nontrivial because it implies that the $\mathrm{RG}$ flow parameter plays the role of a dynamical time. There are several arguments suggesting this connection, at least close to a fixed point of the RG flow - a fixed point being an on-shell or vacuum geometry in string theory. In the world-sheet sigma model for the on-shell or fixed point geometry, it is easy to construct generic operators that cause excitations which are both tachyonic and as well, relevant perturbations resulting in RG flows. An example where the tachyonic excitation is marginal appears in [1] (page 6) where one considers a target space $M=M_{1}^{d} \times R^{9-d, 1}$, such that the world-sheet sigma model on $M$ is a $C F T$ (of the form $C F T_{1}+C F T_{2}$ ). One can construct composite operators in the sigma model on $M$ comprising of operator $O_{1}$ from $C F T_{1}$ and $O_{2}$ from $C F T_{2}$. In [1], a composite operator is chosen such that it is a marginal operator in the full CFT, the resultant excitation is tachyonic, and $O_{1}$ is a relevant perturbation of $C F T_{1}$ causing an RG flow starting from $M_{1}$. There is therefore a link between tachyonic instability of $M$ (which could lead to processes such as tachyon condensation) and instability of $M_{1}$ with respect to an RG flow (due to the perturbation caused by $O_{1}$ ).

Tachyon condensation can occur both as an on-shell process and through off-shell interactions. One example of the change in geometry caused due to tachyon condensation is the orbifold flow $C / Z_{n} \rightarrow C$ - this is studied as an on-shell process in [2] and as an off-shell process in, for example, [3]. There exists a solution to an RG flow that describes this change in geometry qualitatively - this exact solution (to the Ricci flow ) first appeared in the mathematics literature in a paper of H-D Cao [4] and has been discussed independently in the physics context by Adams, Polchinski and Silverstein [2] (the exact solution also appears in [5]). This example has led to the use of RG flow to qualitatively study tachyon condensation.

These results strongly suggest a link between (in)stability of a vacuum geometry with respect to a suitable RG flow and its off-shell (in)stability 2 . Motivated by this, we propose an investigation of off-shell stability of vacua

\footnotetext{
${ }^{2}$ In fact, the orbifold flow example and ideas in [1] suggest that (in)stability with respect to RG flow may give an approximate indication of on-shell (in)stability as well.
} 
in closed string theory by studying their stability with respect to various RG flows. The study of off-shell stability of vacua using string field theory techniques is a hard problem. The study of stability of a geometry with respect to an RG flow, while not an easy problem, is mathematically well-posed in many cases. It may offer insights in a target space, rather than world-sheet picture, of the types of instabilities of string vacua. The world-sheet RG flows of closed string theory are geometric flows, the simplest of these (truncated to first order in $\alpha^{\prime}$ ) being the Ricci flow. The Ricci flow is a subject of active research in mathematics and has been used to prove the Poincaré conjecture (for papers related to this, see [6], [7]). There are also stability results for various geometries with respect to Ricci flow (we discuss some of these in section II). Thus, there is an excellent opportunity to generalize these techniques (as well as techniques from relativity) to address stability of geometries with respect to world-sheet RG flows 3 . The stability analysis for various geometries in relativity can be adapted to study stability with respect to geometric flows as well. A stability analysis of special solutions (such as the hairpin brane) to RG flows of Dirichlet sigma models has been done recently in [9].

One of the most well-studied vacuum geometries in string theory is of the form $A d S_{p} \times S^{q}$ obtained as a stationary point of a supergravity action (for specific $p$ and $q$ ) — an investigation of its stability with respect to RG flow would therefore be interesting 4 . Ideally the stability of $A d S_{p} \times S^{q}$ ought to be studied with respect to an RG flow for which it is a fixed point. However, we recall that the curvature of $A d S_{p} \times S^{q}$ is usually sourced by the RR field. Although there are world-sheet actions with a component that is the sigma model on $A d S_{p} \times S^{q}$ (see section II for a review), there exists no computation of a $\beta$ function with an RR field. $A d S_{3}$ is a fixed point of $\mathrm{RG}$ flow with a NS-NS $B$ field [12]. However this construction does not generalize to $A d S$ space in higher dimensions.

In this paper, as a first step, we address the stability of $n$ dimensional (Euclidean) AdS space (hyperbolic space $\mathbf{H}^{n}$ ) with respect to RG flow 5 . The $\mathrm{RG}$ flow we choose is the Ricci flow. AdS space expands uniformly under

\footnotetext{
${ }^{3}$ It has even been suggested in $[8$ that the thermodynamic instability of a black hole may be related to its instability under Ricci flow.

${ }^{4}$ Classical stability of $A d S_{p} \times M^{q}$ geometries appearing as Freund-Rubin compactifications has been studied in [10].

${ }^{5}$ For a complete discussion of classical stability of $A d S$ spacetime in the context of relativity, see [11.
} 
Ricci flow. One can study if perturbations of $A d S$ space decay, and if the perturbed geometry approaches $A d S$ space under Ricci flow (up to overall scale). This notion of stability is called geometric stability of $A d S$ space with respect to Ricci flow. We prove geometric stability of $A d S_{n}$ with respect to Ricci flow for perturbations obeying specific asymptotic behaviour. The techniques we use are motivated by recent work of Andersson and Moncrief [13] for compact hyperbolic space in the context of relativity - these can be generalized to study noncompact spaces. The world-sheet sigma model with $A d S$ target space is not a CFT, however a stability result with respect to Ricci flow should be indicative of stability results with respect to RG flows with additional fields (for which $A d S_{n}$ may be a fixed point). The $A d S_{n}$ sigma model was also studied by Friess and Gubser [14], who obtained the $\beta$ function in a particular large $n$ expansion. We comment in the last section of the paper on some curious connections between our result and theirs. The techniques we use to investigate stability with respect to Ricci flow can be generalized to RG flow with a $B$ field. Throughout the paper, we assume that the truncation of the $\mathrm{RG}$ flow to first order (in $\alpha^{\prime}$ ) is a valid approximation. When the curvature of the geometry is of order $1 / \alpha^{\prime}$, the sigma model perturbation theory breaks down. In this case, other effects, such as those caused by winding tachyons become important. In the context of asymptotically $A d S$ spaces, this is discussed in [15].

The plan of the paper is as follows: Section II contains a summary, for physicists, of a set of techniques to investigate stability of fixed points of $\mathrm{RG}$ flows. One of the techniques is used in the mathematics of Ricci flow to investigate stability of compact manifolds. We outline another technique which can be applied to investigate stability of noncompact manifolds and then describe the study of geometric stability of $A d S_{n}$ using these techniques. Finally we discuss the motivations for such a study from string theory. This section summarizes the results and the steps involved in proving them, for readers who may want to skip the mathematical details.

Section III and the Appendices contain the main results, proofs, a discussion on boundary terms, asymptotic conditions on the perturbation, and the associated mathematical details. Section IV is a discussion of future projects and some connections with results in [14]. 


\section{Stability techniques}

The Ricci flow arises in physics as the simplest lowest-order (in square of string length $\alpha^{\prime}$ ) RG flow of the world-sheet sigma model for closed strings. In this context, the Ricci flow is the flow of the metric of the target space with respect to the $\mathrm{RG}$ flow parameter $t$. Both in physics and mathematics, we are interested in a flow of geometries (i.e., of metrics mod diffeomorphisms). Therefore all flows related to each other by $t$ dependent diffeomorphisms generated by a vector field $V$ are equivalent; we write the generic flow in this class — the Ricci-de Turck flow as:

$$
\frac{\partial g_{i j}}{\partial t}=-\alpha^{\prime}\left(R_{i j}+\nabla_{i} V_{j}+\nabla_{j} V_{i}\right)
$$

Ricci flow in two and three dimensions on compact manifolds is now well-understood - with curvature conditions on an initial geometry, much is known about the limiting geometry under Ricci flow [16, 17]. This is not the case for solutions to Ricci flow in higher dimensions, or on noncompact manifolds. Stability results for geometries under small perturbations are therefore useful in such cases. An innovative stability technique due to Cao, Hamilton and Ilmanen [18] applies monotonicity formulae under Ricci flow derived by Perelman [6] (the $\lambda$ functional and shrinker entropy). The $\lambda$ functional, for instance, is defined as follows: First, consider a compact manifold $M$ with metric $g$. Define the functional

$$
\mathcal{F}(g, f):=\int_{M} e^{-f}\left(|\nabla f|^{2}+R\right) d V
$$

Then, the $\lambda$ functional is defined by

$$
\lambda(g):=\inf \left\{\mathcal{F}(g, f): f \in C_{c}^{\infty}(M), \int e^{-f} d V=1\right\}
$$

and has some nice properties. Under Ricci flow, $\lambda(g)$ is constant only on fixed points of geometry 6 and is monotonically increasing otherwise. As shown in [18], these properties can then be used to investigate linear stability of a fixed point $g$. Consider a perturbation at $t=0, g^{p}(0)=g(0)+h(0)$. Let $\lambda(g)=c$.

\footnotetext{
${ }^{6}$ These geometries, which are fixed points of Ricci-de Turck flow, are called steady Ricci solitons. It can be shown that the only steady Ricci solitons on compact manifolds are Ricci flat.19.
} 
This is constant along Ricci flow. Let $\lambda\left(g^{p}(0)\right)$ be the value of the $\lambda$ functional for the perturbed geometry at $t=0$. If $\lambda\left(g^{p}(0)\right)-c>0$, then clearly $\lambda\left(g^{p}(t)\right)$ can only increase for $t>0$. So the perturbed geometry will not approach the fixed point. The sign of $\lambda\left(g^{p}(0)\right)-c$ can therefore be used to determine stability of the fixed point. This is evaluated in a linearized approximation where $h(0)$ is assumed small, and it gives a notion of linear stability when the sign is negative. A similar analysis works for the shrinker entropy, which is constant on shrinking Ricci solitons (i.e., geometries that shrink uniformly under Ricci-de Turck flow), and is monotonically increasing otherwise. The shrinker entropy can be used to determine 'geometric stability' of shrinking Ricci solitons. Consider a perturbation of a shrinking Ricci soliton. If the perturbed geometry approaches that of the shrinking Ricci soliton under Ricci-de Turck flow (up to overall scale), we say the soliton is geometrically stable. Using the shrinker entropy, it is shown in [18] that $\mathbf{S}^{n}$ is geometrically stable. For results on stability analysis of other geometries, see [18], [20], [21], 22]. A discussion of stability of Einstein metrics under Ricci flow appears in [23]. A stability result for flat space under Ricci flow is derived in [24]. The linear stability of compact hyperbolic space under (a normalized) Ricci flow is discussed in the appendix to [25]. For a generalization of the stability analysis of [18] to RG flow with a $B$ field, see [26].

It is interesting to note that in the context of relativity, a paper by Fischer and Moncrief that appeared earlier (than [6]) uses closely related stability techniques to analyze stability of certain Einstein metrics [27]. The authors of 27] construct a monotonic quantity in time (the reduced Hamiltonian) that is constant only on a class of self-similar solutions to the Einstein equation. The reduced Hamiltonian can be used to obtain linear stability results as in the case of Ricci flow.

An 'expander entropy' similar to the shrinker entropy, for expanding Ricci solitons has been constructed in [28]. The $\lambda$ functional and these entropies are defined on compact manifolds, and cannot be generalized easily to noncompact manifolds. It is possible that such entropies could be defined with some curvature conditions on the geometry under the flow - for an example on asymptotically flat spaces with negative curvature, see [29]. However, if we wish to examine stability of noncompact manifolds in general, we need other techniques. In the context of relativity, stability of compact hyperbolic space has been examined by Andersson and Moncrief [13] - the techniques used can be generalized to study noncompact manifolds. They can also be adapted to study stability with respect to geometric flows like the Ricci flow. 
We will outline the techniques we use in this paper, which are motivated by those in [13. Start with a fixed point geometry $g$ under a geometric flow. Consider a perturbed geometry $g^{p}(t)=g(t)+h(t)$. We assume $h(t)$ is small so that we can work with a linearized flow equation for $h$. We then define a particular Sobolev norm of the perturbation $h$ given in terms of integrals of the perturbation and its spatial derivatives as defined in Eq.(III.24). One of the main tasks is to bound the Sobolev norm under the linearized flow and prove that the Sobolev norm goes to zero as $t \rightarrow \infty$. This can be done by defining some integrals we call 'energies' — which are given by the squared $L^{2}$ norms of the perturbation and its derivatives with respect to $t$ (see Eq. (III.12)). We first show the energies decay under the flow and then use this to prove that the Sobolev norm decays under the flow. In many cases, there are results generically termed Sobolev inequalities or Sobolev embeddings that then imply that the perturbation and its derivatives decay pointwise everywhere on the manifold. This gives a linear stability result for the fixed point $g$ with respect to the geometric flow. On noncompact manifolds, we may need to prescribe asymptotic behaviour for the perturbation and its derivatives to deal with boundary terms that arise while studying the evolution of the Sobolev norm. We prescribe the asymptotic behaviour at initial $t$ and check that this asymptotic form of the perturbation is preserved by the flow equation.

We can apply this technique to prove geometric (linear) stability of a geometry such as Euclidean $A d S_{n}\left(\mathbf{H}^{n}\right)$ with respect to Ricci flow. We first study its linear stability with respect to the flow Eq. (III.8), chosen so that $\mathbf{H}^{n}$ is a fixed point. The solutions to Eq. (III.8) are related to those of Ricci flow with a rescaled metric and flow parameter. The linear stability of $\mathbf{H}^{n}$ with respect to Eq. (III.2) implies geometric linear stability with respect to Ricci flow. The linear stability of $\mathbf{H}^{n}$ with respect to the flow given by Eq. (III.2) is proved as we have outlined, by showing that the Sobolev norm of the perturbation is bounded under the flow.

As discussed in the introduction, the main motivation is a better understanding of the off-shell stability of $A d S_{n}$ through an investigation of its stability with respect to a suitable $\mathrm{RG}$ flow. There are various sigma model descriptions of $A d S$ space. $A d S_{3}$ is a fixed point of the RG flow of the sigma model with metric and NS-NS field (with the $H$ field proportional to the volume form) [12]. $A d S_{3} \times S^{3} \times T^{4}$ is obtained as a near-horizon limit of the sigma model with a $B$ field describing the NS-5 brane (this is an exact solution in heterotic string theory) [30]. The sigma model with $A d S_{3} \times S^{3}$ target 
space has been studied in [31, for example. The sigma model with $A d S_{n}$ target space has been analyzed in [14]. There is a sigma model description of $A d S_{5} \times S^{5}$ as well, by Metsaev and Tseytlin [32]. They propose a world-sheet action that accounts for the RR field as well; and whose bosonic part is the usual sigma model on $A d S_{5} \times S^{5}$. However, there is no computation of a $\beta$ function - they provide arguments for why the action should be conformal. Since an RG flow with an RR field is not known, one cannot study the stability of $A d S_{5} \times S^{5}$ using sigma model RG flows with this geometry as fixed point. However, one can still choose an RG flow such as Ricci flow, with respect to which this geometry evolves, and analyze geometric stability. This should be indicative of the stability under metric perturbations with respect to other RG flows as well.

We conclude this section by remarking that it may also be possible to generalize (classical) stability calculations in relativity and supergravity to study stability of a wider class of geometries under RG flows. For example, Gibbons and Hartnoll [33] discuss classical stability of a class of geometries (that include some higher dimensional black holes) under certain types of perturbations. They show that the stability under these perturbations is related to the spectrum of the Lichnerowicz laplacian on a lower dimensional manifold, the 'base' manifold. However, unlike this classical stability analysis, we cannot gauge away the trace of a perturbation when studying stability under RG flows.

There has been extensive work on studying classical perturbations of $A d S$ spacetime itself. For example, in [11], it is shown that the equations governing scalar, vector and tensor perturbations of $A d S$ spacetime all reduce to the action of a positive, symmetric, elliptic differential operator on a space of square-integrable functions. These equations can be solved with appropriate boundary conditions (the perturbations vanish at the conformal boundary). This then implies that $A d S$ spacetime is classically linearly stable. Further, given an assumption on the solutions to the linearized Einstein equation for $A d S$ spacetime - namely that (up to infinitesimal diffeomorphisms) they are uniquely determined by their series expansion at the conformal boundary - it is possible to obtain certain global uniqueness results for the $A d S$ spacetime [34]. For a proof that the above assumption holds for conformally compact Riemannian-Einstein metrics, see [35].

In the stability problem we discuss in this paper, the geometric PDE governing the evolution of perturbations of (Euclidean) AdS space is the linearized Ricci flow, not the linearized Einstein equation. Nevertheless, it 
would be desirable to solve the linear stability problem of $A d S$ space under Ricci flow completely, with suitable boundary conditions, rather than the analysis based on the Sobolev norms, and the asymptotic behaviour we have imposed. It is also possible that a local existence result can be obtained for the linearized flow for initial data in some appropriate Sobolev space. We hope to return to this question in future work.

\section{Linearized analysis of stability of hyper- bolic space}

The conventions we follow in the paper for the curvature tensors are those of Wald [36].

We investigate the stability of $n$ dimensional hyperbolic space $\mathbf{H}^{n}$ with respect to the Ricci flow

$$
\frac{\partial \tilde{g}_{i j}}{\partial \tilde{t}}=-\alpha^{\prime} \tilde{R}_{i j}
$$

$\tilde{t}$ is the RG flow parameter.

$\mathbf{H}^{n}$ is not a fixed point of the Ricci flow. It is convenient to first study its stability with respect to a flow with $\mathbf{H}^{n}$ as fixed point, such that its solutions are related to those of Ricci flow. We consider the flow

$$
\frac{\partial g_{i j}}{\partial t}=-\alpha^{\prime}\left[R_{i j}+c g_{i j}\right]
$$

whose solutions are related to those of (III.1) by

$$
\begin{gathered}
\tilde{t}=\frac{1}{\alpha^{\prime} c} e^{\alpha^{\prime} c t}, \\
\tilde{g}_{i j}=e^{\alpha^{\prime} c t} g_{i j} .
\end{gathered}
$$

Recall that the Riemann tensor of $\mathbf{H}^{n}$ is

$$
R_{i j k l}=-\frac{1}{a}\left[g_{i k} g_{j l}-g_{i l} g_{j k}\right],
$$

where $a>0$ is a constant. Therefore,

$$
\begin{gathered}
R_{i j}=-\frac{(n-1)}{a} g_{i j}, \\
R=-\frac{n(n-1)}{a} .
\end{gathered}
$$


$\mathbf{H}^{n}$ is a fixed point of Eq. (II.2) with $c=\frac{(n-1)}{a}$.

We now wish to consider perturbations of the metric $g$ of $\mathbf{H}^{n}$. The perturbed metric $g_{i j}^{p}=g_{i j}+h_{i j}$. We will study the evolution of the perturbation under the flow Eq.(III.2) (more precisely, a flow related to it by diffeomorphisms) in a linearized approximation. We will then show in the rest of the section that the perturbation decays under the flow. $g_{i j}^{p}(t)$ is related to a solution of Ricci flow (in the linearized approximation) by the rescalings Eq.(III.3). The solution to Ricci flow is $\tilde{g}_{i j}(\tilde{t})=e^{\alpha^{\prime} c t}\left(g_{i j}+h_{i j}(t)\right)$; and if we show that $h_{i j}(t) \rightarrow 0$ as $t \rightarrow \infty$, then the perturbed geometry approaches that of $\mathbf{H}^{n}$ under Ricci flow in this approximation, up to the overall scale $e^{\alpha^{\prime} c t}$. This is our precise notion of geometric linear stability.

Under the flow Eq.(III.2), in a linearized approximation, the perturbation $h_{i j}$ obeys

$$
\frac{\partial h_{i j}}{\partial t}=\frac{\alpha^{\prime}}{2}\left[\left(\Delta_{L} h\right)_{i j}+\nabla_{i} \nabla_{j} H-\nabla_{i}\left(\nabla^{k} h_{k j}\right)-\nabla_{j}\left(\nabla^{k} h_{k i}\right)-2 c h_{i j}\right] .
$$

Here and in what follows, all covariant derivatives are taken with respect to the background metric $g . H=g^{i j} h_{i j}$ is the trace of the perturbation.

$$
\left(\Delta_{L} h\right)_{i j}=\Delta h_{i j}+2 R_{k i l j} h^{k l}-R_{i}^{k} h_{j k}-R_{j}^{k} h_{i k}
$$

is the Lichnerowicz laplacian acting on symmetric 2-tensors (all curvature tensors being those of the background metric).

A $t$ dependent diffeomorphism generated by vector field $X$ modifies the flow (III.2) for the metric $g^{p}$ to

$$
\frac{\partial g_{i j}^{(p)}}{\partial t}=-\alpha^{\prime}\left[R_{i j}^{(p)}+c g_{i j}^{(p)}+\nabla_{i}^{(p)} X_{j}+\nabla_{j}^{(p)} X_{i}\right]
$$

where (III.8) is now written in the new coordinates 7 .

$X$ can be chosen so that when we now linearize (III.8), we can eliminate the 'divergence' terms that appear in the linearized flow Eq.(III.6), $\frac{\alpha^{\prime}}{2}\left(\nabla_{i} \nabla_{j} H-\nabla_{i}\left(\nabla^{k} h_{k j}\right)-\nabla_{j}\left(\nabla^{k} h_{k i}\right)\right)$, leading to the simplified flow (in the new coordinates) 8

$$
\frac{\partial h_{i j}}{\partial t}=\frac{\alpha^{\prime}}{2}\left[\left(\Delta_{L} h\right)_{i j}-2 c h_{i j}\right] .
$$

\footnotetext{
${ }^{7}$ We can relate solutions to this modified flow with solutions to an appropriate Ricci -de Turck flow of the form Eq.(II.1) by rescalings as in Eq.(III.3).

${ }^{8}$ This is part of the 'de-Turck trick' in Ricci flow used to prove short-time existence of solutions. See page 79, 16, for details.
} 
Denote

$$
\frac{\partial h_{i j}}{\partial t}=(\Lambda h)_{i j}
$$

where

$$
\begin{aligned}
& (\Lambda h)_{i j}=\frac{\alpha^{\prime}}{2}\left[\left(\Delta_{L} h\right)_{i j}-2 c h_{i j}\right] \\
& =\frac{\alpha^{\prime}}{2}\left[\Delta h_{i j}-\frac{2}{a} H g_{i j}+\frac{2}{a} h_{i j}\right] .
\end{aligned}
$$

Now, it can be easily checked that when the background metric is that of $\mathbf{H}^{n}$, the operator $\Lambda$ acting on the 'divergence' part of the perturbation preserves this form. Such terms can be removed by a suitable $t$ dependent diffeomorphism as we did before. In the rest of the paper, we therefore only consider divergence-free (i.e., transverse) perturbations. Such a perturbation $h_{i j}$ satisfies $\nabla^{j} h_{i j}=0$.

Let us now consider the following general 'energy integral' $E^{(K)}$, defined by

$$
E^{(K)}=\int_{M}\left|\left(\Lambda^{(K)} h\right)_{i j}\right|^{2} d V
$$

where the notation $\left(\Lambda^{(2)} h\right)_{i j}=(\Lambda \Lambda h)_{i j}$, for example $\left(\right.$ denote $\left.\left(\Lambda^{(0)} h\right)_{i j}=h_{i j}\right)$. Further, we define the 'cross term'

$$
E^{(K, K+1)}=\int_{M}\left(\Lambda^{(K+1)} h\right)^{i j}\left(\Lambda^{(K)} h\right)_{i j} d V
$$

We will bound $E^{(K)}$ and $E^{(K, K+1)}$ under the flow. In order to do this, we need the following lemmas:

Proposition 3.1: If $h_{i j}$ is a transverse perturbation, then $\left(\Lambda^{(K)} h\right)_{i j}$ is transverse on $\mathbf{H}^{n}$.

Proof: Consider a transverse tensor $T_{i j}$ (i.e., $\nabla^{j} T_{i j}=0$ ). Let $\operatorname{Tr} \operatorname{T}$ denote $g^{i j} T_{i j}$. Then

$$
\nabla^{j}(\Lambda T)_{i j}=\frac{\alpha^{\prime}}{2} \nabla^{j}\left[\Delta T_{i j}-\frac{2}{a}(\operatorname{Tr} T) g_{i j}+\frac{2}{a} T_{i j}\right]
$$




$$
\begin{aligned}
\nabla^{j}(\Delta T)_{i j}= & \Delta\left(\nabla^{j} T_{i j}\right)+g^{p k} g^{j s}\left[\nabla_{p}\left(R_{s k i}{ }^{m} T_{m j}+R_{s k j}{ }^{m} T_{i m}\right)+\right. \\
& \left.R_{s p k}{ }^{m} \nabla_{m} T_{i j}+R_{s p i}{ }^{m} \nabla_{k} T_{m j}+R_{s p j}{ }^{m} \nabla_{k} T_{i m}\right] \\
= & \frac{2}{a} \nabla_{i}(\operatorname{Tr} T) .
\end{aligned}
$$

Therefore, it follows from (III.14) and (III.15) that $\nabla^{j}(\Lambda T)_{i j}=0$. Setting $T_{i j}=h_{i j},(\Lambda h)_{i j}$ is transverse, and it follows that $\left(\Lambda^{(K)} h\right)_{i j}$ is transverse for all positive integers $K$.

Lemma 3.2: For a transverse symmetric tensor $T_{i j}$, we have the following inequality on $\mathbf{H}^{n}$ :

$$
\begin{gathered}
\int_{M}\left(\Delta T_{i j}\right) T^{i j} d V \leq \frac{1}{a} \int_{M}(\operatorname{Tr} T)^{2} d V-\frac{n}{a} \int_{M}\left|T_{i j}\right|^{2} d V \\
+\int_{\partial M}\left[\left(\nabla_{k} T_{i j}\right)-\left(\nabla_{j} T_{i k}\right)\right] n^{k} T^{i j} d A
\end{gathered}
$$

Proof: Consider the manifestly nonnegative quantity $\epsilon$ :

$$
\begin{gathered}
\epsilon=\int_{M}\left|\nabla_{k} T_{i j}-\nabla_{j} T_{i k}\right|^{2} d V \\
=2 \int_{M}\left|\nabla_{k} T_{i j}\right|^{2} d V-2 \int_{M}\left(\nabla_{k} T_{i j}\right)\left(\nabla^{j} T^{i k}\right) d V
\end{gathered}
$$

Now,

$$
\begin{aligned}
& \int_{M}\left(\nabla_{k} T_{i j}\right)\left(\nabla^{j} T^{i k}\right)=\int_{\partial M}\left(\nabla_{k} T_{i j}\right) T^{i k} n^{j} d A \\
& -\int_{M} R_{j k i m} T^{m j} T^{i k} d V-\int_{M} R_{k}^{m} T_{i m} T^{i k} d V
\end{aligned}
$$

where we integrated by parts, commuted two covariant derivatives, and used the fact that $T$ is a transverse tensor.

On $\mathbf{H}^{n}$, we therefore have

$$
\int_{M}\left(\nabla_{k} T_{i j}\right)\left(\nabla^{j} T^{i k}\right)=\int_{\partial M}\left(\nabla_{k} T_{i j}\right) T^{i k} n^{j} d A-\frac{1}{a} \int_{M}(\operatorname{Tr} T)^{2} d V+\frac{n}{a} \int_{M}\left|T_{i k}\right|^{2} d V
$$


Similarly,

$$
\int_{M}\left|\nabla_{k} T_{i j}\right|^{2} d V=\int_{\partial M}\left(\nabla_{k} T_{i j}\right) T^{i j} n^{k} d A-\int_{M}\left(\Delta T_{i j}\right) T^{i j} d V
$$

Therefore, from (III.18 III.20),

$$
\begin{gathered}
\epsilon=2 \int_{\partial M}\left(\nabla_{k} T_{i j}\right) T^{i j} n^{k} d A-2 \int_{M}\left(\Delta T_{i j}\right) T^{i j} d V \\
-2 \int_{\partial M}\left(\nabla_{k} T_{i j}\right) T^{i k} n^{j} d A+\frac{2}{a} \int_{M}(\operatorname{Tr} T)^{2} d V-\frac{2 n}{a} \int_{M}\left|T_{i k}\right|^{2} d V
\end{gathered}
$$

Since $\epsilon \geq 0$, the inequality in the lemma follows.

We are now ready to prove the following bound for the energies:

\section{Theorem 3.3: On $\mathbf{H}^{n}$,}

$$
\frac{d E^{(K)}}{d t} \leq-\alpha^{\prime} \frac{(n-2)}{a} E^{(K)}+\alpha^{\prime} \int_{\partial M}\left[\left(\nabla_{k} T_{i j}\right)-\left(\nabla_{j} T_{i k}\right)\right] n^{k} T^{i j} d A
$$

where, in the inequality above (and in the proof), $T_{i j}=\left(\Lambda^{(K)} h\right)_{i j}$.

\section{Proof:}

$$
\begin{aligned}
\frac{d E^{(K)}}{d t}= & 2 \int_{M}\left(\Lambda^{(K)} h\right)^{i j}\left(\Lambda^{(K+1)} h\right)_{i j} d V \\
= & \alpha^{\prime} \int_{M} T^{i j}\left[\Delta T_{i j}-\frac{2}{a}(\operatorname{Tr} T) g_{i j}+\frac{2}{a} T_{i j}\right] d V \\
\leq & -\alpha^{\prime} \frac{(n-2)}{a} \int_{M} T^{i j} T_{i j} d V-\alpha^{\prime} \frac{1}{a} \int_{M}(\operatorname{Tr} T)^{2} d V+ \\
& \alpha^{\prime} \int_{\partial M}\left[\left(\nabla_{k} T_{i j}\right)-\left(\nabla_{j} T_{i k}\right)\right] n^{k} T^{i j} d A \\
\leq & -\alpha^{\prime} \frac{(n-2)}{a} E^{(K)}+ \\
& \alpha^{\prime} \int_{\partial M}\left[\left(\nabla_{k} T_{i j}\right)-\left(\nabla_{j} T_{i k}\right)\right] n^{k} T^{i j} d A
\end{aligned}
$$


where the inequality in the last two steps follows from Proposition 3.1 and Lemma 3.2 .

We note that for $n=2$, we only need to consider the flow of the trace of the perturbation. We can construct 'energies' as above from the $t$ derivatives of the trace and bound them under the flow. This is discussed in Appendix D. In fact, we can prove that the trace decays pointwise on the manifold, and this is enough to prove geometric linear stability of $\mathbf{H}^{2}$.

We now define a particular Sobolev norm of the perturbation (see Appendix B for a discussion of Sobolev norms), denoted by $\|h\|_{k, 2}$, by the following equation:

$$
\begin{aligned}
\left(\|h\|_{k, 2}\right)^{2}= & \int_{M}\left|h_{i j}\right|^{2} d V+\int_{M}\left|\nabla_{p_{1}} h_{i j}\right|^{2} d V+\ldots \\
& \ldots+\int_{M}\left|\nabla_{p_{1}} \ldots \nabla_{p_{k}} h_{i j}\right|^{2} d V
\end{aligned}
$$

In the above expression, $\left|h_{i j}\right|^{2}$, for example, is the square of the (pointwise) tensor norm of the perturbation, i.e., $h^{i j} h_{i j}$, and indices are raised with respect to the background metric $g$. We assume that this norm is defined for our perturbation at initial $t$.

From Lemma A1.1 in the Appendix, the square of the above Sobolev norm can be written in a form more useful for our purposes (for $k$ an even positive integer) and $\left\{A_{i}\right\}$ for $i=1, . ., k$ constants:

$$
\begin{aligned}
\left(\|h\|_{k, 2}\right)^{2}= & A_{0} \int_{M}\left|h_{i j}\right|^{2} d V+A_{1} \int_{M} h^{i j} \Delta h_{i j} d V+A_{2} \int_{M}\left|\Delta h_{i j}\right|^{2} d V \ldots . \\
& \ldots .+A_{k} \int_{M}\left|\Delta^{\frac{k}{2}} h_{i j}\right|^{2} d V \\
& + \text { (similar sum over integrals of traces of above tensors) } \\
& + \text { b.t. }
\end{aligned}
$$

$b . t$ is an abbreviation for boundary terms. When $k$ is odd, we have, similarly,

$$
\begin{aligned}
&\left(\|h\|_{k, 2}\right)^{2}= A_{0} \int_{M}\left|h_{i j}\right|^{2} d V+A_{1} \int_{M} h^{i j} \Delta h_{i j} d V+\ldots \ldots \\
& \ldots .+A_{k} \int_{M}\left(\Delta^{\frac{(k-1)}{2}} h^{i j}\right)\left(\Delta^{\frac{(k+1)}{2}} h_{i j}\right) d V \\
&+(\text { similar sum over integrals of traces of above tensors) } \\
&+ \text { b.t. } \\
& \text { (III.26) }
\end{aligned}
$$


The boundary terms that appear in the discussion above as well as everywhere else in the paper are discussed in Appendix C (we will impose appropriate asymptotic behaviour on the perturbations later so that the boundary terms vanish). By Corollary A1.3, we can study the evolution of $\left(\|h\|_{k, 2}\right)^{2}$ under the flow by studying the evolution of the integral $\int_{M}\left(\Lambda^{P} h\right)^{i j}\left(\Lambda^{Q} h\right)_{i j} d V$ for arbitrary integers $P, Q \geq 0$. This task is made easier by the following Proposition;

Proposition 3.4: Let $P, Q \geq 0$ be two integers such that $(P-Q)=R \geq 0$. Let $T_{i j}=\left(\Lambda^{Q} h\right)_{i j} \cdot \int_{M}\left(\Lambda^{P} h\right)^{i j}\left(\Lambda^{Q} h\right)_{i j} d V=\int_{M} T^{i j}\left(\Lambda^{R} T\right)_{i j} d V$.

(i) If $R$ is odd, then

$$
\int_{M} T^{i j}\left(\Lambda^{R} T\right)_{i j} d V=\int_{M}\left(\Lambda^{\frac{(R-1)}{2}} T\right)^{i j}\left(\Lambda^{\frac{(R+1)}{2}} T\right)^{i j} d V+\text { b.t. }
$$

(ii) If $R$ is even, then

$$
\int_{M} T^{i j}\left(\Lambda^{R} T\right)_{i j} d V=\int_{M}\left(\Lambda^{\frac{R}{2}} T\right)^{i j}\left(\Lambda^{\frac{R}{2}} T\right)^{i j} d V+\text { b.t. }
$$

Proof: From Eq.(III.11),

$$
\begin{gathered}
\left(\Lambda^{R} T\right)_{i j}=\frac{\alpha^{\prime}}{2}\left[\Delta\left(\Lambda^{(R-1)} T\right)_{i j}-\frac{2}{a} \operatorname{Tr}\left(\Lambda^{(R-1)} T\right) g_{i j}+\frac{2}{a}\left(\Lambda^{(R-1)} T\right)_{i j}\right] \\
\int_{M} T^{i j} \Delta\left(\Lambda^{(R-1)} T\right)_{i j} d V=\int_{M} \Delta T^{i j}\left(\Lambda^{(R-1)} T\right)_{i j} d V+\text { b.t, }
\end{gathered}
$$

by integrating by parts. Therefore it follows from Eq.(III.29) and Eq.(III.30) that

$$
\left.\int_{M} T^{i j}\left(\Lambda^{R} T\right)_{i j} d V=\int_{M}(\Lambda T)^{i j} \Lambda^{(R-1)} T\right)_{i j} d V+\text { b.t, }
$$

and that we can iterate this procedure until we get the righthand sides of Eq.(III.27) and Eq.(III.28). 
Corollary 3.5 : Thus, modulo boundary terms, from Corollary A1.3 and Proposition 3.4, it follows that $\left(\|h\|_{k, 2}\right)^{2}$ can be written as a sum over integrals which are precisely the energies $E^{(K)}$ and 'cross terms' $E^{(K, K+1)}$ that we defined earlier.

We now discuss the asymptotic behaviour that is required of the perturbation for the various boundary terms (listed in Appendix C) to vanish. We would like to impose such asymptotic behaviour at some $t=t_{0}$ and then argue that it is preserved by the flow at all $t>t_{0}$. To do this, it is convenient to start with the Poincaré ball metric for $\mathbf{H}^{n}$ - i.e., $\mathbf{H}^{n}$ is the interior of the n-dimensional unit ball with metric $g=e / \rho^{2}$ where $e$ is the Euclidean metric. Choosing Cartesian coordinates $\left\{x^{i}\right\}$,

$$
d s^{2}=\frac{1}{\rho^{2}} \sum_{i=1}^{n}\left(d x^{i}\right)^{2} .
$$

$\rho=\left(1-r^{2}\right) /(2 \sqrt{a}) . \quad r=\sqrt{\sum_{i=1}^{n}\left(x^{i}\right)^{2}}$. The volume element $d V=\rho^{-n} d x^{n}$. The geodesic distance $R(x)$ from the origin to $x$ is

$$
R=\sqrt{a} \ln \left(\frac{1+r}{1-r}\right)
$$

Then, asymptotically as $r \rightarrow 1, \rho \approx(1-r) /(\sqrt{a})$, and $\exp (-R /(\sqrt{a})) \approx$ $\frac{1}{2}(1-r)$. We restrict ourselves in the rest of the paper to perturbations for which, as $r \rightarrow 1, h_{j k} \sim O\left((1-r)^{\beta}\right)$ implies $\partial_{i} h_{j k} \sim O\left((1-r)^{\beta-1}\right)$. Then, a study of the boundary terms listed in Appendix $\mathrm{C}$ reveals that the asymptotic behaviour required for the boundary terms to vanish is $h_{j k} \sim O\left((1-r)^{\frac{n-4}{2}+\epsilon}\right)$, $\epsilon>0$. We can impose such a condition at some $t=t_{0}$. We can then check whether the evolution operator $\Lambda$ preserves this asymptotic behaviour. Since $h_{j k}\left(t_{0}\right) \sim O\left((1-r)^{\frac{n-4}{2}+\epsilon}\right)$ as $r \rightarrow 1$, it is easily seen (because the Poincaré ball metric is conformal to the Euclidean metric) that $(\Lambda h)_{j k} \sim O\left((1-r)^{\frac{n-4}{2}+\epsilon}\right)$ asymptotically. Therefore, studying Eq.(III.11) as $r \rightarrow 1$, it is clear that $h_{i j}(t)$ obeys this asymptotic behaviour for $t>t_{0}$. Due to the (simple) nature of the evolution operator $\Lambda$, it is probable that a more rigorous local existence result can be obtained by starting with an initial condition $h \in H_{s}^{2}\left(T_{2} M, g\right) \sqrt{9}$ and adapting some of the results in [37] to the flow equation Eq.(III.11).

\footnotetext{
${ }^{9}$ See Appendix B for the definition of the Sobolev space $H_{s}^{2}\left(T_{2} M, g\right)$.
} 
We observe that for $n \geq 4$, the asymptotic behaviour required is a 'falloff', and corresponds to the perturbation falling off exponentially as a function of the geodesic distance from the origin $R(r)$ as $r \rightarrow 1$. The asymptotic conditions that characterize asymptotically anti-de Sitter spaces also correspond to exponential fall-offs in the geodesic distance from the origin. However, these are obtained in different coordinates, in for e.g., [38] and a comparison of the two sets of asymptotic behaviour is not straightforward, due to the fact that the two boundaries have different topologies.

Corollary 3.6 : For solutions $h_{i j}(t)$ of Eq.(III.11) that obey the asymptotic behaviour $h_{j k}(t) \sim O\left((1-r)^{\frac{n-4}{2}+\epsilon}\right)$ as $r \rightarrow 1$,

(i) It follows from Theorem 3.3 that

$$
E^{(K)}(t) \leq E^{(K)}\left(t_{0}\right) e^{-D\left(t-t_{0}\right)},
$$

where $D=\alpha^{\prime}(n-2) / a$.

(ii) The cross term $E^{(K, K+1)}(t)$ obeys the inequality

$$
E^{(K, K+1)}(t) \leq \sqrt{E^{(K)}(t) E^{(K+1)}(t)} \leq \sqrt{E^{(K)}\left(t_{0}\right) E^{(K+1)}\left(t_{0}\right)} e^{-D\left(t-t_{0}\right)}
$$

This follows from the Cauchy-Schwarz inequality for vector spaces with inner product $\langle.,$.$\rangle and norm \|\|,.\langle T, W\rangle \leq\|T\|\|W\|$, when we consider the $L^{2}$ norm of tensors.

(iii) A consequence of (i), (ii) and Corollary 3.5 is that the Sobolev norm $\|h\|_{k, 2}$ is bounded with respect to the flow Eq.(III.11), and $\|h\|_{k, 2}(t) \rightarrow 0$ as $t \rightarrow \infty$.

We now use certain Sobolev-type inequalities derived in the context of $\mathbf{H}^{n}$ by L. Andersson [39]. Sobolev inequalities are generically inequalities between various norms (a useful reference for Sobolev inequalities for domains in $\mathbf{R}^{n}$ is [40 ). We are particularly interested in a Sobolev inequality that bounds the $C^{k}$ norm of the perturbation from above by a constant times its Sobolev norm (see Appendix B for the definition of the $C^{k}$ norm). The Sobolev inequalities in 39] for $\mathbf{H}^{n}$ are obtained by first showing that the Sobolev norm on $\mathbf{H}^{n}-$ i.e., on the unit ball with metric $g=e / \rho^{2}$ is equivalent to a weighted Sobolev 
norm on the unit ball with metric $e$ with suitable weights functions which are powers of $\rho$ (see Appendix B for definition of a weighted Sobolev norm). The result most useful for us from [39] is the following inequality for $s>n / 2$ ( $s$ is a nonnegative integer):

$$
\|h\|_{C^{k}} \leq C\|h\|_{k+s, 2} .
$$

$C$ is a constant. We have already seen that $\|h\|_{k+s, 2}$ (the Sobolev norm of the perturbation of $\mathbf{H}^{n}$ ) is bounded under the flow Eq. (III.11) and decays exponentially as $t \rightarrow \infty$. Therefore, choosing $s$ large enough, inequality Eq.(III.36) implies that the perturbation and its derivatives up to order $k$ are bounded pointwise on $M$, and decay as $t \rightarrow \infty$. Thus $\mathbf{H}^{n}$ is linearly stable with respect to the flow Eq.(III.11) for perturbations obeying the asymptotic conditions we have prescribed. Further, we know from Eq.(III.3) that such perturbations of $\mathbf{H}^{n}$ also decay under Ricci flow and the geometry approaches that of $\mathbf{H}^{n}$ up to an overall scale — so this implies geometric linear stability for $\mathbf{H}^{n}$ with respect to Ricci flow.

\section{Discussion}

From the discussion so far, we conclude that $A d S_{n}$ and $\mathbf{S}^{n}$ are geometrically (linearly) stable with respect to Ricci flow. The stability of $A d S_{p} \times S^{q}$ with respect to Ricci flow still remains to be investigated - this problem is not straightforward since hyperbolic space expands under Ricci flow while the sphere contracts. However, we at least know that this geometry is stable when the (metric) perturbation is restricted to either $A d S_{p}$ or $S^{q}$. A stability result with respect to Ricci flow is indicative of a stability result with respect to other RG flows, when restricted to only metric perturbations. In the absence of an RG flow involving the RR field, the only other computation we can now attempt is to check stability of $A d S_{3}$ with respect to RG flow with a $B$-field. It should be possible to do this computation by generalizing the energies defined in section III and using similar techniques. If $A d S_{3}$ is stable with respect to this $\mathrm{RG}$ flow (restricting to only metric perturbations) as we expect, this would offer yet another reason why $A d S$ space is interesting in string theory. It would also be indicative of off-shell stability (with respect to some processes at least) in string field theory.

What does this stability result for $A d S_{n}$ imply for its quotients? In three dimensions, one of the quotients of $A d S$ space is a black hole geometry - 
the BTZ black hole [41]. The stability result for $A d S_{n}$ required a careful analysis of boundary terms and an imposition of asymptotic behaviour on the perturbations. Since the BTZ black hole is obtained from $A d S_{3}$ by global identifications, this analysis will be different for the BTZ black hole, and thus its stability remains an open question.

Is it possible to generalize this result on linear stability to a notion of nonlinear stability? We use a linearized flow and therefore require the perturbation $h(t)$ to be small. The linear stability results of [18] (on compact manifolds) with respect to Ricci flow, on the other hand, only require the perturbation to be small at some initial $t$. The diffeomorphism-invariant functionals and entropies then allow a study of the perturbed geometry with respect to the (full nonlinear) Ricci flow. We would like to generalize the linear stability result in this paper to a result where we only require the perturbation to be small at initial $t$ - this is a notion of nonlinear stability, since the perturbed geometry is evolving under the nonlinear flow. We therefore need to either extend or find an analogue of Perelman's entropies for noncompact manifolds - this may be possible with some curvature conditions on the geometry under the flow. In the context of relativity, Andersson and Moncrief prove nonlinear stability of compact hyperbolic space in this sense [13] - hopefully these techniques can be generalized to study nonlinear stability of $\mathbf{H}^{n}$ with respect to the geometric flows considered in this paper.

Finally, we comment on a curious (possibly coincidental) connection to a study of the $A d S_{n}$ sigma model by Friess and Gubser [14]. These authors observe that the effective expansion parameter in the sigma model is $\kappa=$ $-\frac{\alpha^{\prime}(n-1)}{a}$. They compute the $\beta$ function of the sigma model in the large $n$ limit but to all orders in $\alpha^{\prime}$ (such that $\frac{\alpha^{\prime}(n-1)}{a}$ is kept fixed). The authors find a nontrivial $A d S_{n}$ fixed point at second order in this expansion for a particular value of $\kappa$. Curiously, the parameter $\kappa$ in [14] is exactly the same as the decay constant characterizing the (exponential) rate of decay of the trace of the perturbation $H=g^{i j} h_{i j}$ in our analysis (see Appendix D for this derivation). We do not know yet of a logical argument connecting these two computations, since ours is a target space analysis, whereas the computation in [14] is done entirely on the world-sheet. 


\section{Acknowledgements}

I am indebted to Vincent Moncrief for introducing me to the stability techniques used in this paper and for various enjoyable discussions at every stage of this project. I thank Lars Andersson for useful comments and for bringing [39] to my attention. I also thank Eric Woolgar for a discussion on asymptotic analysis and for comments on a draft of the paper. I wish to acknowledge other comments on this work and related topics by Ioannis Bakas, Huai-Dong Cao, Viqar Husain, K Narayan, Todd Oliynyk and Bala Sathiapalan. This work was initiated during a visit to Albert Einstein Institute, Golm and I thank the institute for hospitality. This work is supported by funds from the Natural Sciences and Engineering Research Council of Canada.

\section{Appendix A: Useful results}

Here we prove some Lemmas required for section 3 of the paper.

Lemma A1.1 : For some choice of constants $\left\{B_{i}\right\}, i=1, \ldots, k$, and $k$ a positive integer, on $\mathbf{H}^{n}$,

(i) When $k$ is even,

$$
\begin{aligned}
\int_{M}\left|\nabla_{p_{1}} \ldots . \nabla_{p_{k}} h_{i j}\right|^{2} d V= & B_{0} \int_{M}\left|h_{i j}\right|^{2} d V+B_{1} \int_{M} h^{i j} \Delta h_{i j} d V+B_{2} \int_{M}\left|\Delta h_{i j}\right|^{2} d V \\
& \ldots .+B_{k} \int_{M}\left|\Delta^{\frac{k}{2}} h_{i j}\right|^{2} d V \\
& +(\text { sum over integrals of traces of above tensors) } \\
& + \text { b.t. }
\end{aligned}
$$


(ii) When $k$ is odd,

$$
\begin{aligned}
\int_{M}\left|\nabla_{p_{1}} \ldots \nabla_{p_{k}} h_{i j}\right|^{2} d V= & B_{0} \int_{M}\left|h_{i j}\right|^{2} d V+B_{1} \int_{M} h^{i j} \Delta h_{i j} d V+\ldots \ldots \\
& \ldots .+B_{k} \int_{M}\left(\Delta^{\frac{(k-1)}{2}} h^{i j}\right)\left(\Delta^{\frac{(k+1)}{2}} h_{i j}\right) d V \\
& +(\text { sum over integrals of traces of above tensors) } \\
& + \text { b.t. }
\end{aligned}
$$

Proof: We prove this result by induction. First note that

$$
\begin{aligned}
\int_{M}\left|\nabla_{p} h_{i j}\right|^{2} d V= & -\int_{M} h^{i j} \Delta h_{i j} d V \\
& +\int_{\partial M} h^{i j}\left(\nabla_{p} h_{i j}\right) n^{p} d A .
\end{aligned}
$$

Similarly,

$$
\begin{aligned}
\int_{M}\left|\nabla_{p} \nabla_{s} h_{i j}\right|^{2} d V= & \int_{\partial M}\left(\nabla_{p} \nabla_{s} h^{i j}\right)\left(\nabla^{s} h_{i j}\right) n^{p} d A-\int_{M} \nabla^{s} h^{i j} \Delta\left(\nabla_{s} h_{i j}\right) d V \\
= & \int_{\partial M}\left(\nabla_{p} \nabla_{s} h^{i j}\right)\left(\nabla^{s} h_{i j}\right) n^{p} d A+\int_{M} \nabla^{s} h^{i j} \nabla_{s}\left(\Delta h_{i j}\right) d V \\
& -\frac{(n-1)}{a} \int_{M}\left|\nabla_{p} h_{i j}\right|^{2} d V-\frac{8}{a} \int_{M}\left(\nabla^{s} h^{i j}\right)\left(\nabla_{i} h_{s j}\right) d V .
\end{aligned}
$$

Now,

$$
\begin{aligned}
-\frac{8}{a} \int_{M}\left(\nabla_{s} h_{i j}\right)\left(\nabla^{i} h^{s j}\right) d V= & -\frac{8}{a} \int_{\partial M}\left(\nabla_{s} h_{i j}\right) h^{s j} n^{i} d A+\frac{8}{a} \int_{M} h^{s j}\left(\nabla^{i} \nabla_{s} h_{i j}\right) d V \\
= & -\frac{8}{a} \int_{\partial M}\left(\nabla_{s} h_{i j}\right) h^{s j} n^{i} d A-\frac{8(n+1)}{a^{2}} \int_{M}\left|h_{s j}\right|^{2} d V \\
& +\frac{16}{a^{2}} \int_{M} H^{2} d V .
\end{aligned}
$$

In the above expression, we commuted two covariant derivatives in the final step and used the expressions for the curvature tensors of the background. Further,

$$
\int_{M} \nabla^{s} h^{i j} \nabla_{s}\left(\Delta h_{i j}\right) d V=\int_{\partial M} \nabla_{s} h^{i j}\left(\Delta h_{i j}\right) n^{s} d A-\int_{M}\left|\Delta h_{i j}\right|^{2} d V .
$$


Using (VI.5) and (VI.6) in (VI.4), we see that the statement of Lemma A1.1 is indeed satisfied.

Now assume the induction hypothesis, i.e., that both parts of the statement of Lemma A1.1 are true for all $\int_{M}\left|\nabla_{p_{1}} \ldots \nabla_{p_{m}} h_{i j}\right|^{2} d V$, and $m \leq k$. Let us first assume $k$ is even. Then, consider $m=(k+1)$. First, let $A_{p_{2} \ldots p_{k} i j}=\nabla_{p_{2}} \ldots \nabla_{p_{k}} h_{i j}$. Then, $\nabla_{p_{1} \ldots .} \nabla_{p_{k}} h_{i j}=\nabla_{p_{1}} A_{p_{2} \ldots p_{k} i j}$.

$$
\begin{aligned}
& \int_{M}\left|\nabla_{q} \nabla_{p_{1} \ldots .} \nabla_{p_{k}} h_{i j}\right|^{2} d V=\int_{M} \nabla^{q} \nabla^{p_{1}} A^{p_{2} \ldots i j} \nabla_{q} \nabla_{p_{1}} A_{p_{2} \ldots i j} d V, \\
= & -\int_{M} \nabla^{p_{1}} A^{p_{2} \ldots i j} \Delta \nabla_{p_{1}} A_{p_{2} \ldots i j} d V+\text { b.t. } \\
= & -\int_{M} \nabla^{p_{1}} A^{p_{2} \ldots j} \nabla_{p_{1}} \Delta A_{p_{2} \ldots j} d V+\frac{(n-1)}{a} \int_{M}\left|\nabla_{p_{1}} A_{p_{2} \ldots i j}\right|^{2} d V \\
+ & \frac{2}{a} \sum_{l=2}^{k} \int_{M} \nabla^{p_{1}} A^{p_{2} . . p_{l} . . j} \nabla_{p_{l}} A_{p_{2} . . . p_{1} . . j} d V-\frac{2}{a} \sum_{l=2}^{k} \int_{M} \nabla^{p_{1}} A^{p_{2 . . j}} \nabla^{m} A_{p_{2} . . m . . j} g_{p_{1} p_{l}} d V \\
+ & \frac{4}{a} \int_{M} \nabla^{p_{1}} A^{p_{2} . . i j} \nabla_{i} A_{p_{2} . . . p_{1 j}} d V-\frac{4}{a} \int_{M} \nabla^{p_{1}} A^{p_{2} \ldots i j} \nabla^{r} A_{p_{2} \ldots . r j} g_{p_{1} i} d V \\
& +b . t
\end{aligned}
$$

Now we look at the structure of each term in the last line of (VI.7). Consider

$$
I_{1}=\int_{M} \nabla^{p_{1}} A^{p_{2} \ldots j} \nabla_{p_{1}} \Delta A_{p_{2} \ldots j} d V=-\int_{M} \Delta A^{p_{2} \ldots j} \Delta A_{p_{2} \ldots j} d V+b . t
$$

Writing $A_{p_{2} \ldots p_{k} i j}=\nabla_{p_{2}} \ldots \nabla_{p_{k}} h_{i j}$ we can again commute $\Delta$ and $\nabla_{p_{2}}$ in the integrand of $I_{1}$. Further, by a sequence of such steps, we can write this term as a sum of $\int_{M} \Delta^{\frac{k}{2}} h^{i j} \Delta^{\frac{(k+2)}{2}} h_{i j} d V$ and similar terms with lower powers of $\Delta$ to which the induction hypothesis applies. This is because every step where the covariant derivatives are commuted produces Riemann curvature terms. In $\mathbf{H}^{n}$, these are simply proportional to products of metric components and produce traces of pairs of derivative operators or of the perturbation. This applies to other terms in the last line of (VI.7) as well. For the term

$$
I_{2}=\frac{(n-1)}{a} \int_{M}\left|\nabla_{p_{1}} A_{p_{2} \ldots i j}\right|^{2} d V
$$


the induction hypothesis applies directly. For

$$
\begin{aligned}
I_{3} & =\int_{M} \nabla^{p_{1}} A^{p_{2} . . p_{l} . . j} \nabla_{p_{l}} A_{p_{2} \ldots p_{1} . . j} d V \\
I_{4} & =\int_{M} \nabla^{p_{1}} A^{p_{2} \ldots j} \nabla^{m} A_{p_{2} \ldots m . . j} g_{p_{1} p_{l}} d V
\end{aligned}
$$

again one can commute derivatives and the argument in the previous paragraph applies.

The terms

$$
\begin{aligned}
I_{5} & =\frac{4}{a} \int_{M} \nabla^{p_{1}} A^{p_{2} \ldots i j} \nabla_{i} A_{p_{2} \ldots p_{1 j}} d V=-\frac{4}{a} \int_{M} \nabla_{i} \nabla^{p_{1}} A^{p_{2} \ldots i j} A_{p_{2} \ldots p_{1} j} d V+b . t \\
I_{6} & =\int_{M} \nabla_{i} A^{p_{2} \ldots i j} \nabla^{r} A_{p_{2} \ldots r j} d V
\end{aligned}
$$

are somewhat different. Since $A_{p_{2} \ldots p_{k} i j}=\nabla_{p_{2}} \ldots \nabla_{p_{k}} h_{i j}$, we can commute derivatives, till we get a term with $\nabla_{i} h^{i j}$ which is zero. Other terms are proportional to the Riemann tensor, as before. Thus it is easy to see that the statement of Lemma A1.1 is true, by these obvious steps. The same can be done if $k$ is odd.

Lemma A1.2 : For $p$ a positive integer, and some constants $c_{K}, K=0, \ldots, p$,

$$
\Delta^{p} h_{i j}=\sum_{K=0}^{p} c_{K}\left(\Lambda^{(K)} h\right)_{i j}+\sum_{K=0}^{p} \tilde{c}_{K} \operatorname{Tr}\left(\Lambda^{(K)} h\right) g_{i j}
$$

We have used the notation $\left(\Lambda^{0} h\right)_{i j}=h_{i j}$ and as usual, $\operatorname{Tr}\left(\Lambda^{(K)} h\right)=\left(\Lambda^{(K)} h\right)_{i j} g^{i j}$.

Proof: The case $p=1$ is obviously true from Eq.(III.11). We can write

$$
\Delta h_{i j}=\frac{2}{\alpha^{\prime}}(\Lambda h)_{i j}+\frac{2}{a} H g_{i j}-\frac{2}{a} h_{i j} .
$$

Now assume the statement of the lemma is true for $\Delta^{p}$ for some $p$. Then it is easy to see that the statement of the lemma is true for $\Delta^{p+1} h_{i j}$. From Eq.(III.11),

$$
\Delta^{p+1} h_{i j}=\frac{2}{\alpha^{\prime}}\left(\Lambda\left(\Delta^{p} h\right)\right)_{i j}+\frac{2}{a}\left(\operatorname{Tr} \Delta^{p} h\right) g_{i j}-\frac{2}{a} \Delta^{p} h_{i j},
$$


and one can then substitute for $\Delta^{p} h_{i j}$ from Eq.(VI.12).

Corollary A1.3 : It therefore follows that an integral of the form $\int_{M}\left|\Delta^{\frac{k}{2}} h_{i j}\right|^{2} d V$ or $\int_{M}\left(\Delta^{\frac{(k-1)}{2}} h^{i j}\right)\left(\Delta^{\frac{(k+1)}{2}} h_{i j}\right) d V$ can be written as a sum of integrals of the form $\int_{M}\left(\Lambda^{P} h\right)^{i j}\left(\Lambda^{Q} h\right)_{i j} d V$ for integers $P, Q \geq 0$

\section{Appendix B: Sobolev and weighted Sobolev spaces}

Consider a manifold $M$ with Riemannian metric $g$. We define Sobolev spaces of sections of tensor bundles over $M$. Let us first recall the definition of various norms. For a section $v$ of $T_{s}^{r} M$ we can define the pointwise tensor norm by using the metric. Denote this by $|v|_{g}$. Define, for $1 \leq p<\infty$,

$$
\|v\|_{L^{p}}=\left(\int_{M}\left(|v|_{g}\right)^{p} d V_{g}\right)^{\frac{1}{p}}
$$

to be the $L^{p}$ norm of $u$ when $\int_{M}\left(|v|_{g}\right)^{p} d V_{g}<\infty$. Define $\|v\|_{\infty}=\inf \{K$ : $|v(x)| \leq K$ almost everywhere on $M\}$. This is also called the essential supremum of $|v|$. Now let $u$ be a section of $T_{s}^{r} M$ of class $C^{k}$. Let $\alpha=$ $\left(\alpha_{1}, \ldots . \alpha_{j}\right)$ be the $j$-tuple of non-negative integers $\alpha_{i}$. We call $\alpha$ a multiindex. $\nabla^{\alpha}=\nabla_{1}^{\alpha_{1}} \ldots \nabla_{j}^{\alpha_{j}}$ is a differential operator of order $|\alpha|=\sum_{i=1}^{j} \alpha_{i}$. The $C^{k}$ norm of u is defined as

$$
\|u\|_{C^{k}}=\max _{0 \leq|\alpha| \leq k} \sup _{x \in M}\left|\nabla^{\alpha} u(x)\right|
$$

Define the Sobolev, or $H_{k}^{p}$ norm as

$$
\|u\|_{k, p}=\left(\sum_{0 \leq|\alpha| \leq k} \int_{M}\left(\left|\nabla^{\alpha} u\right|_{g}\right)^{p} d V_{g}\right)^{\frac{1}{p}},
$$

if $1 \leq p<\infty$. Also define $\|u\|_{k, \infty}=\max _{0 \leq|\alpha| \leq k}\left\|\nabla^{\alpha} u\right\|_{\infty}$.

The Sobolev space $\left.H_{k}^{p}\left(T_{s}^{r} M, g\right)\right)$ is the completion of $\left\{u\right.$ a section of $T_{s}^{r} M$ of class $\left.C^{k}:\|u\|_{k, p}<\infty\right\}$ with respect to the norm $\|.\|_{k, p}$. We shall abbreviate this in the paper as $H_{k}^{p}$ wherever it is obvious that we are dealing with tensors on $M . H_{k}^{2}\left(T_{s}^{r} M, g\right)$ is a Hilbert space. 
Define now the weighted Sobolev norm of $u$ with weight functions $w_{\alpha}$ as

$$
\|u\|_{k, p, w}=\left(\sum_{0 \leq|\alpha| \leq k} \int_{M}\left(\left|\nabla^{\alpha} u\right|_{g}\right)^{p} w_{\alpha} d V_{g}\right)^{\frac{1}{p}},
$$

if $1 \leq p<\infty$. The weighted Sobolev spaces are defined using this norm, similar to Sobolev spaces.

\section{Appendix C: Boundary terms}

We list the various types of boundary terms that arise in the analysis of the stability of $\mathbf{H}^{n}$.

Let $T_{i j}=\left(\Lambda^{(K)} h\right)_{i j}$.

The boundary terms associated with the proof of Lemma 3.2 are of the form

$$
B_{1}=\int_{\partial M}\left[\left(\nabla_{k} T_{i j}\right)-\left(\nabla_{j} T_{i k}\right)\right] n^{k} T^{i j} d A .
$$

The boundary terms associated with the proof of Proposition 3.4 are of the form

$$
\begin{aligned}
& B_{2}=\int_{\partial M} T^{i j} \nabla_{p}\left(\Lambda^{(Q)} h\right)_{i j} n^{p} d A . \\
& B_{3}=\int_{\partial M} \nabla_{p} T^{i j}\left(\Lambda^{(Q)} h\right)_{i j} n^{p} d A .
\end{aligned}
$$

The boundary terms arising from the proof of Lemma A1.1 are of the form

$$
\begin{aligned}
& B_{4}=\int_{\partial M} \nabla^{p_{1}} \ldots(\Delta)^{R} . . \nabla^{p_{k}} h^{i j} \nabla_{s} \nabla_{p_{1}} . .(\Delta)^{Q} . . \nabla_{p_{k}} h_{i j} n^{s} d A . \\
& B_{5}=\int_{\partial M} \nabla^{p_{1}} \ldots(\Delta)^{R} . . \nabla^{p_{k}} h^{s j} \nabla_{s} \nabla_{p_{1}} . .(\Delta)^{Q} . . \nabla_{p_{k}} h_{i j} n^{i} d A .
\end{aligned}
$$

$(\Delta)^{2} A_{i_{1} \ldots i_{k}}=\Delta \Delta A_{i_{1} \ldots i_{k}}$ according to our notation. The asymptotic behaviour required to eliminate these boundary terms is given in section III. 


\section{Appendix D: Flow of the trace of the per- turbation}

Taking the trace of Eq.(III.9) with respect to the background metric, we get the flow of the trace of the perturbation,

$$
\frac{\partial H}{\partial t}=\frac{\alpha^{\prime}}{2}[\Delta H-2 c H]=L H .
$$

We now define the energy integral,

$$
E_{H}^{(K)}=\int_{M}\left(L^{(K)} H\right)^{2} d V
$$

where $L^{(2)} H=L L H$. We can study the flow of $E_{H}^{(K)}$.

$$
\begin{aligned}
\frac{\partial E_{H}^{(K)}}{\partial t}= & 2 \int_{M} L^{(K)} H \frac{\partial L^{(K)} H}{\partial t} d V \\
= & \alpha^{\prime} \int_{M} L^{(K)} H[\Delta-2 c] L^{(K)} H d V \\
= & -2 c \alpha^{\prime} E_{H}^{(K)}-\alpha^{\prime} \int_{M}\left|\nabla_{i}\left(L^{(K)} H\right)\right|^{2} d V+ \\
& \alpha^{\prime} \int_{\partial M}\left(L^{(K)} H\right) \nabla_{i}\left(L^{(K)} H\right) n^{i} d A, \\
\leq & -2 \frac{\alpha^{\prime}(n-1)}{a} E_{H}^{(K)}+\alpha^{\prime} \int_{\partial M}\left(L^{(K)} H\right) \nabla_{i}\left(L^{(K)} H\right) n^{i} d A .
\end{aligned}
$$

The asymptotic behaviour discussed in section III guarantees that the boundary term in (IX.3) vanishes. Then we can reproduce the rest of the analysis - we can construct the Sobolev norm of $H$, argue using the energy bound (IX.3) that this norm decays under the flow of the trace (IX.1), and that $H$ decays pointwise on $M$. Clearly the decay of $H$ must be faster than or equal to $C e^{-\left(\alpha^{\prime}(n-1) / a\right) t}$, where $C$ is some constant. Note that the rate of exponential decay governed by $-\alpha^{\prime}(n-1) / a$ is the same as the constant $\kappa$ in [14].

\section{References}

[1] M Headrick, S Minwalla, T Takayanagi, Class Quant Grav 21 (2004) S1539. 
[2] A Adams, J Polchinski, E Silverstein, JHEP 0110 (2001) 029.

[3] Y Okawa, B Zwiebach, JHEP 0403 (2004) 056.

[4] H-D Cao, J Diff Geom 45 (1997) 257.

[5] M Gutperle, M Headrick, S Minwalla, V Schomerus, JHEP 0301 (2003) 073.

[6] G Perelman, arXiv: math/0211159, math/0303109, math/0307245.

[7] H-D Cao, X-P Zhu, Asian Journal of Mathematics 10 (2006) 165; J Morgan, G Tian, Ricci flow and the Poincare Conjecture, Clay Math Monographs Volume 3, CMI, 2007.

[8] M Headrick, T Wiseman, Class.Quant.Grav. 23 (2006) 6683. See also G. Holzegel, T. Schmelzer, C. Warnick, Class Quant Grav 24 (2007) 6201 .

[9] I Bakas, C Sourdis, arXiv:0704.3985.

[10] O DeWolfe, DZ Freedman, SS Gubser, GT Horowitz, I Mitra, Phys Rev D65 (2002) 064033; SS Gubser, I Mitra, JHEP 0207 (2002) 044; T Shiromizu, D Ida, H Ochiai, T Torii, Phys Rev D64 (2001) 084025.

[11] A Ishibashi, RM Wald, Class Quant Grav 21 (2004) 2981.

[12] G Horowitz, D Welch, Phys Rev Lett 71 (1993) 328.

[13] L Andersson, V Moncrief, Einstein spaces as attractors for the Einstein flow, unpublished.

[14] J Friess, S Gubser, Nucl Phys B750 (2006) 111.

[15] GT Horowitz, E Silverstein, Phys.Rev. D73 (2006) 064016.

[16] B Chow, The Ricci flow: an introduction, Mathematical Surveys and Monographs, Volume 110, AMS, 2004.

[17] P. Topping, Lectures on the Ricci flow, London Mathematical Society lecture note series 325, Cambridge University Press, 2006.

[18] H-D Cao, R Hamilton, T Ilmanen, arXiv: math/0404165. 
[19] J-P Bourguignon in Global differential geometry and global analysis, Lectures in Mathematics 838, ed. D Ferus, Springer, 1981.

[20] C Guenther, J Isenberg, D Knopf, Comm Anal Geom 10 (2002) no.4, 741; Int. Math. Res. Not. (2006), Article ID 96253, doi: 10.1155/IMRN/2006/96253.

[21] N Sesum, arXiv: math/0410062.

[22] X Dai, X Wang, G Wei, arXiv: math/0504527.

[23] R Ye, Trans Amer Math Soc 338 (1993) 871.

[24] O Schnuerer, F Schulze, M Simon, Comm Anal Geom 16 (2008) 127.

[25] D Knopf, A Young, Proc. Amer. Math. Soc., to appear.

[26] T Oliynyk, V Suneeta, E Woolgar, Nucl Phys B739 (2006) 441.

[27] AE Fischer, V Moncrief, Class Quant Grav 19 (2002) 5557.

[28] M Feldman, T Ilmanen, L Ni, arXiv: math/0405036.

[29] T Oliynyk, V Suneeta, E Woolgar, Phys Lett B610 (2005) 115.

[30] M Cvetic, AA Tseytlin, Phys Lett B366 (1996) 95.

[31] A Giveon, D Kutasov, N Seiberg, Adv Theor Math Phys 2 (1998) 733.

[32] RR Metsaev, AA Tseytlin, Nucl Phys B533 (1998) 109.

[33] GW Gibbons, SA Hartnoll, Phys Rev D66 (2002) 064024; see also GW Gibbons, SA Hartnoll, CN Pope, Phys Rev D67 (2003) 084024.

[34] MT Anderson, Class Quant Grav 23 (2006) 6935.

[35] MT Anderson, M Herzlich, J Geom Phys, 58, (2008), 179.

[36] RM Wald, General Relativity, The University of Chicago Press, 1984.

[37] ME Taylor, Partial Differential Equations vol. 3: Nonlinear equations, Springer, New York, 1996.

[38] M Henneaux, C Teitelboim, Comm Math Phys 98, no. 3 (1985 ) 391. 
[39] L Andersson, Indiana U. Math. J. 42, no.4 (1993) 1359.

[40] RA Adams, JJF Fournier, Sobolev spaces, 2nd Ed., vol. 140, Pure and Applied Mathematics Series, Academic Press, 2003.

[41] M Banados, C Teitelboim, J Zanelli, Phys Rev Lett 69 (1992) 1849; M Banados, M Henneaux, C Teitelboim, J Zanelli, Phys Rev D48 (1993) 1506. 